\title{
Cubesat Antenna Analysis and Design: Are you using the right computational electromagnetic tool?
}

\author{
Ibtissam Latachi', Tajjeedine Rachidi ${ }^{2}$, and Mohammed Karim ${ }^{1}$ \\ ${ }^{1}$ Université Sidi Mohammed Ben Abdellah, Faculty of Sciences Dhar El Mehraz, Fez, Morocco \\ ${ }^{2} \mathrm{Al}$ Akhawayn University in Ifrane (AUI), Ifrane, Morocco \\ Corresponding author: Ibtissam Latachi (e-mail: latachi.ibtissam@gmail.com).
}

\begin{abstract}
Antenna systems play a critical role in establishing wireless communication links and sustaining remote sensing requirements for Cubesat applications. In addition to the usual antenna design requirements, Cubesat-based spacecrafts impose additional stringent constraints related to the on-board available space, power consumption and development costs. To develop optimal antenna prototypes while considering all these constraints and decrease trial and error related costs, computational electromagnetics (CEM) simulation tools are used. The accuracy of simulation results depends to a great extent on the choice of the appropriate CEM tool for the particular antenna problem to be analyzed; ergo, identifying and answering key questions about design objectives and requirements is necessary for informed decisionmaking throughout the selection and design processes. However, this could be quite challenging because of existing gaps both in the practitioners' knowledge about different CEM tools capabilities, limitations, and design know-how. This is especially true for non-specialists such as students and academics involved in student driven Cubesat projects. Therefore, the rationale of this manuscript is to bridge those gaps and clarify some common misconception commonly encountered during the selection and design processes. In that regard, first, an overview of existing antenna configurations commonly used in Cubesat communications is provided. Next, antenna design general workflow is presented. Then, capabilities and limitations of different CEM solving methods are presented. After that, CEM software selection process trade-offs and possible sources of errors are discussed from a practical viewpoint. Finally, a case study of Masat-1 antenna system design is presented as practical example.
\end{abstract}

INDEX TERMS Antenna, CEM software, CEM solving methods, Computational electromagnetics, Cubesat, Simulation

\section{INTRODUCTION}

D eveloping antenna systems within the Cubesat paradigm not only opens the door for many new applications, but also introduces new challenges; the antenna design is ruled by communication requirements (frequency band, high gain, low loss, coverage, ...), Cubesat platform constraints (size, deployment, attitude constraints ...), and/or mission aspects, compelling designers to devise feasible and optimized antenna systems that fit in tiny, stowable packages; Therefore, recent years have witnessed an ever-increasing demand for computational tools for antenna design and analysis. With the advent of computing technologies, significant improvements of existing numerical algorithms have been made, and more powerful general purposes software packages have been developed. We distinguish commercial and Open source Computational Electromagnetics (CEM) simulation software. Academic/Inhouse CEM software also exist; however, they are often special purpose software, and their use is restricted to the institution's research community where they were developed, hence they are out of the scope of this paper.

CEM simulation tools play a crucial role in closing the gap between theory and experiment; they help practitioners improve their understanding of the problem in hand and enable them to design efficient and cost-effective prototype. This benefits greatly Cubesat developers who are compelled to develop reliable antenna systems while respecting predefined requirements, imposed by the Cubesat Design Standards (CDS), and stringent financial budgets. Good engineering practice dictates that relevant and recent trends of antenna system development should be surveyed first. Once an appropriate antenna configuration has been selected, a preliminary antenna specifications document is defined. Thereafter, one has to raise and answer some key questions to choose the adequate CEM tools and to make educated decisions during the modeling and analysis processes. Indeed, depending on the supported solving method used to solve Maxwell equations, each CEM software may be best 
suited for a specific category of problems and totally unfit for others. Hence, CEM software have different computing capabilities and choosing the wrong tool can either generate incorrect results or results which take excessively long to compute. Therefore, the main focus of this work is to discuss the main capabilities and limitations of popular CEM methods and highlight possible sources of errors and discrepancies when using CEM software during modeling and numerical validation processes of the antenna model. Needless to say, no single CEM method/software can analyze all types of antenna problems. Also, considering the complete scope of available CEM software, providing comprehensive solutions to all antenna modeling problems in this paper is an unrealistic endeavor. Instead, we aim to provide practical tips to choose the appropriate CEM tools/software for the targeted problem and clarify some common misconceptions encountered during the design process, so that Cubesat developer and engineers can make informed decisions; thus, in this paper, we shall answer the following questions:

- What are the existing types of antenna used in Cubesat communication?

- How to simulate an antenna problem using CEM tools?

- Given the chosen antenna configuration, which CEM solver is better fit to use during simulation and optimization processes?

- What criteria should be considered when selecting CEM software?

- What are the sources of erroneous/inaccurate results? And what are possible causes of discrepancies between various CEM software?

\section{ANTENNA SYSTEMS FOR CUBESAT COMMUNICATIONS}

In order to design an antenna configuration suitable for a Cubesat, the entire mission objectives, and many other constraints must be considered. Table I outlines some of the major design challenges of Cubesat antenna systems. Factors like operating frequencies, data rate, and communication link types supported - whether Telemetry, Tracking and Command (TTC) links, high speed links or inter-satellites links - help define the type and required characteristics of the antenna. Currently, three different antenna types have been considered for Cubesats missions, namely omnidirectional low gain antennas, high gain antennas, and directive selfsteering antennas.

\section{A. OMNIDIRECTIONAL LOW GAIN ANTENNA}

They are recommended for TTC communications because easy link establishment regardless of the satellite attitude is highly required. They typically involve dipoles and monopoles because they are simple to construct, and full spherical radiation is easily achieved when combining several antennas. They are especially used for VHF and UHF links; to reach higher frequency bands, such as $\mathrm{S}, \mathrm{X}, \mathrm{Ku}$, or Ka bands, for TTC links, microstrip antennas and helices are used [1]. Furthermore, monopoles and dipoles are easily stowable within the Cubesat volume thanks to sophisticated deployment mechanisms. In [2], the authors proposed an improved version of the tape-spring model traditionally used to deploy wire antennas; a composite bistable tape springs have been used to fabricate dipoles for improved flexibility. After deployment, they are usually placed on the external face of the CubeSat, allowing space for other electronics. Further studies comparing the performance of different wire antennas are offered in [3] [4].

Table I. Cubesat antenna system design challenges [1]

\begin{tabular}{ll}
\hline \multicolumn{1}{c}{ Design challenges } & \multicolumn{1}{c}{ Rationale } \\
\hline Small size and mass, & $\begin{array}{l}\text { - Low cost budget for the space mission } \\
\text { low power, and easy } \\
\text { low-cost construction } \\
\text { Cubesat standard }\end{array}$ \\
& $\begin{array}{l}\text { - No domination of satellite profile, hence } \\
\text { allowing easy integration of solar panels. }\end{array}$ \\
\hline $\begin{array}{l}\text { Reliability, mechanical } \\
\text { robustness and material } \\
\text { resilience to space } \\
\text { environment }\end{array}$ & $\begin{array}{c}\text { - Resistance to environmental effects, such as } \\
\text { launch } \\
\text { cosmic and Van Allen belt radiations, deep } \\
\text { dielectric charging, differential surface } \\
\text { charging, and material outgassing. }\end{array}$ \\
\hline
\end{tabular}

Electromagnetic compatibility with other - The satellite structure and the components satellite components and among antennas fitted in the same Cubesat structure onboard radiate too. Reduce the resulting mutual coupling, which can cause satellite performance deterioration.

\begin{tabular}{ll}
\hline Impedance matching & $\begin{array}{l}\text { - Power transfer maximization and signal } \\
\text { reflection reduction. }\end{array}$ \\
\hline $\begin{array}{l}\text { High gain and wide } \\
\text { bandwidth }\end{array}$ & $\begin{array}{l}\text { - Enhancement of space-to-earth link budget } \\
\text { - Enabling inter-satellites communications. }\end{array}$ \\
\hline $\begin{array}{l}\text { Frequency and } \\
\text { polarization re- } \\
\text { configurability }\end{array}$ & $\begin{array}{l}\text { - Enabling radiation of more patterns at different } \\
\text { frequencies and polarization }\end{array}$ \\
\hline & - Polarization mismatch elimination. \\
& $-\begin{array}{l}\text { Enhancing system performance and saving } \\
\text { power by directing the antenna beam to desired } \\
\text { direction }\end{array}$ \\
Beam steer-ability & Enabling inter-satellite communications \\
& - Ensuring better coverage.
\end{tabular}

\section{B. HIGH GAIN ANTENNA}

They are mainly used in high-speed and inter-satellite links, where high data rates are favored to transmit bulk data. They are also required for radar and remote sensing applications. They include reflector and reflectarray antennas, horn antennas, and planar antennas. The major challenge of high gain antenna design is stowage restrictions due to highly constrained volume; given the size available in Cubesats, several deployment mechanisms for larger antennas have 
Table II. Pros and cons of some high gain antenna configurations derived from [9]

\begin{tabular}{|c|c|c|c|c|}
\hline & \multicolumn{2}{|c|}{ Small stowage volume ( $<0.1$ Unit) } & \multicolumn{2}{|c|}{ Large stowage volume ( $>0.5$ Unit) } \\
\hline & Patch array & Reflect array & Mesh reflector & Inflatable \\
\hline Pros & $\begin{array}{l}+ \text { Stowage efficiency } \\
+ \text { Non deployable } \\
+ \text { High reliability } \\
+ \text { Low cost } \\
+ \text { Low Side Lob Level } \\
\quad(\text { SLL) }\end{array}$ & $\begin{aligned}+ & \text { Excellent stowage } \\
+ & \text { Simple deployment } \\
& \text { mechanism } \\
+ & \text { Low cost } \\
+ & \text { Low SLL }\end{aligned}$ & $\begin{array}{l}\text { + Good efficiency } \\
+ \text { Large bandwidth } \\
+\sim 1 \mathrm{~m}^{2} \text { aperture }\end{array}$ & $\begin{array}{l}+ \text { Storage efficiency } \\
+ \text { Large bandwidth } \\
+1 \mathrm{~m}^{2} \text { aperture } \\
+ \text { Simple deployment } \\
\quad \text { mechanism }\end{array}$ \\
\hline Cons & $\begin{array}{l}\text { - Feed loss limits gain } \\
\text { - Feed leakage } \\
\text { - Max aperture }<\text { side of } \\
\text { Cubesat }\end{array}$ & $\begin{array}{l}-\quad<50 \% \text { efficiency } \\
- \text { Max aperture less } \\
\text { than } 1 \mathrm{~m}^{2} \\
- \text { Narrow bandwidth }\end{array}$ & $\begin{array}{l}\text { - }>1.5 \text { Unit stowage } \\
- \text { Mechanical complexity } \\
- \text { High SLL } \\
- \text { Cost }\end{array}$ & $\begin{array}{l}\text { - Poor surface accuracy } \\
\text { - Low frequency } \\
\text { - Reliability risk } \\
\text { - High SLL }\end{array}$ \\
\hline
\end{tabular}

been investigated in the literature for each antenna type [1-8]. Table II enumerates their pros and cons.

There are two main types of reflector antennas: off axis reflectors and axisymetric (focal feed, Cassegrain and Gregorian).The simplest is the focal feed where the feed is located at the focal point of the reflector, whereas Cassegrain and Gregorian reflectors add a second concave or convect reflector before or just in front of the focal point.

Reflector antennas provide high gain and good resolution but at the cost of increased mechanical complexity. To overcome this challenge, mesh and inflatable reflectors are preferred over rigid antenna. In many cases, umbrella-like technique is used to deploy meshed reflectors; a metallic mesh surface stretched between a discrete number of parabolic ribs to enable stowage within a size of $0.5 \mathrm{U}$ [9] [10]. For optimal accuracy and performance, a throughout analysis of the mesh surface must be carried out [10].

To further reduce stowage volume, inflatable mechanisms are used; "The basic idea is to implement the antenna on a fabric-like material. This would allow the antenna to be easily folded and stowed in a compact volume. The stowage volumes for the membrane antenna and inflatable antenna are $2 U$ and less than $0.6 U$, respectively" [11].

Reflectarrays are also commonly used because they are lightweight and can be easily integrated with the CubeSat structure, thanks to their flat solar panels which can be folded and stowed to yield stowage efficiency [8][12]. However, reflect-arrays have narrow bandwidth.

Horn antennas are also a viable option for CubeSat communications at higher frequencies because they provide good gain with low cross polarization and low back-lobes. They can be used as standalone antenna but are commonly used as a primary feed for reflector and reflect array antennas. Rahmat-Samii and coworkers [9] [11] propose using a multi-flare horn to feed a Cassegrain reflector system operating at $\mathrm{Ka}$ band for Earth science and deep space missions. Reference [13] readdress optimization of horn antenna as high-performance feeds in reflectors using a novel approach; direct enhancement of the over-all system performance by co-simulating the feed and the reflector during each evaluation. A descriptive summary of reflector and reflector-array antennas analysis and synthesis techniques along with feeding systems can be found in [7] [14].

On the other hand, planar antennas are non-deployable hence they are low profiled antenna with relative ease of manufacturing. They include patch antennas and slot antennas [15]. S-band planar inverted-F antenna arrays were described in [16] and [17]. However, traditional patch antennas vie with solar panels for Cubesat Surface. Thereby, novel approaches for antenna integration with solar panels have been proposed. A patch antenna with high optical transparency can be placed directly on top of solar cells [18]. Development of transparent antenna is based on transparent metal conductors or a conductive wire mesh which is mounted on a transparent substrate material like glass or quartz. Non-transparent antennas are also used; they are typically placed beneath the solar panels. Reference [19] propose a solution based on cavity backed slot antennas circularly polarized operating in UHF band.

\section{DIRECTIVE SELF-STEERING ANTENNA}

They are required in inter-satellite links for various reasons; due to limitations in the accuracy of the Cubesat attitude control subsystem, the antenna must provide a wide bandwidth and a circular polarization which is independent of the spacecraft attitude. Moreover, in the absence of knowledge of the position of the other spacecraft, beamsteering capabilities are a must. Hence, directive selfsteering, such as planar phased antenna array and slot antenna, are typically used [20] [21].

In summary, Cubesat antenna implementation, whether deployable or fixed, must be performed with extra care for 
efficient integration; inappropriate selected antenna or deployment mechanism might hinder other integrated and deployable components, as it might result in stowage volume overuse. Therefore, to avoid such design complications, Computational Electromagnetic (CEM) simulation tools are commonly used by designers during early antenna/Cubesat design stages.

\section{ANTENNA DESIGN FOR CUBESAT APPLICATIONS: CHOOSING THE RIGHT CEM SOFTWARE AND SOLVING METHODS}

\section{A. CEM SOL VING METHODS}

The main purpose of electromagnetic solvers is to compute approximations to Maxwell equations given a set of boundaries and initial conditions. As illustrated in Fig. 1, there are two major categories: full-wave methods and High-Frequency approximation techniques. Depending on the complexity, geometry type (i.e. 2D, 2D-axisymmetric or 3D) and size of the antenna problem subject to analysis, the adequate CEM solving method shall be selected. The problem's complexity depends on the following elements:

- Materials characteristics: Non-linear/Linear; Inhomogeneous; Anisotropic; Dispersive; Lossy; Penetrable, etc.

- Wide-band/Narrow-band applications; Transient/
(PO) and uniform theory of diffraction (UTD) approximations. On the other hand, Full-wave methods are more suitable for problems where the antenna structure size is no more than a few tens of wavelengths.

Since the sizes of antennas used onboard Cubesats are in the order of a few wavelengths, full-wave methods are usually chosen. Therefore, in the remaining of this paper, the main focus is put on full-wave methods commonly used in Computational Electromagnetics (CEM). Full-wave methods include integral-equation and differential-equation approaches. In Table III, we highlight capabilities and limitations of each method.

Integral equation approaches are considered for open problems with two-dimensional field sources; only surface meshing of the electromagnetic field sources is required. Therefore, these methods are recommended for the analysis of antenna with 'planar' geometry. Method Of the Moments (MOM) is an integral-equation technique. Dense linear systems are generated and invoke $\mathrm{O}\left(\mathrm{n}^{2}\right)$ memory to store and $\mathrm{O}\left(\mathrm{n}^{3}\right)$ to be computed. Therefore, analysis of large sized, non-linear or inhomogeneous structures is extremely difficult using MOM method. The latter is highly suited for conductive thin wires (i.e. radius $\sim$ wavelength/1000) or planar homogeneous antenna. To compensate MOM drawbacks, Multilevel Fast Multipole Method (MLFMM)

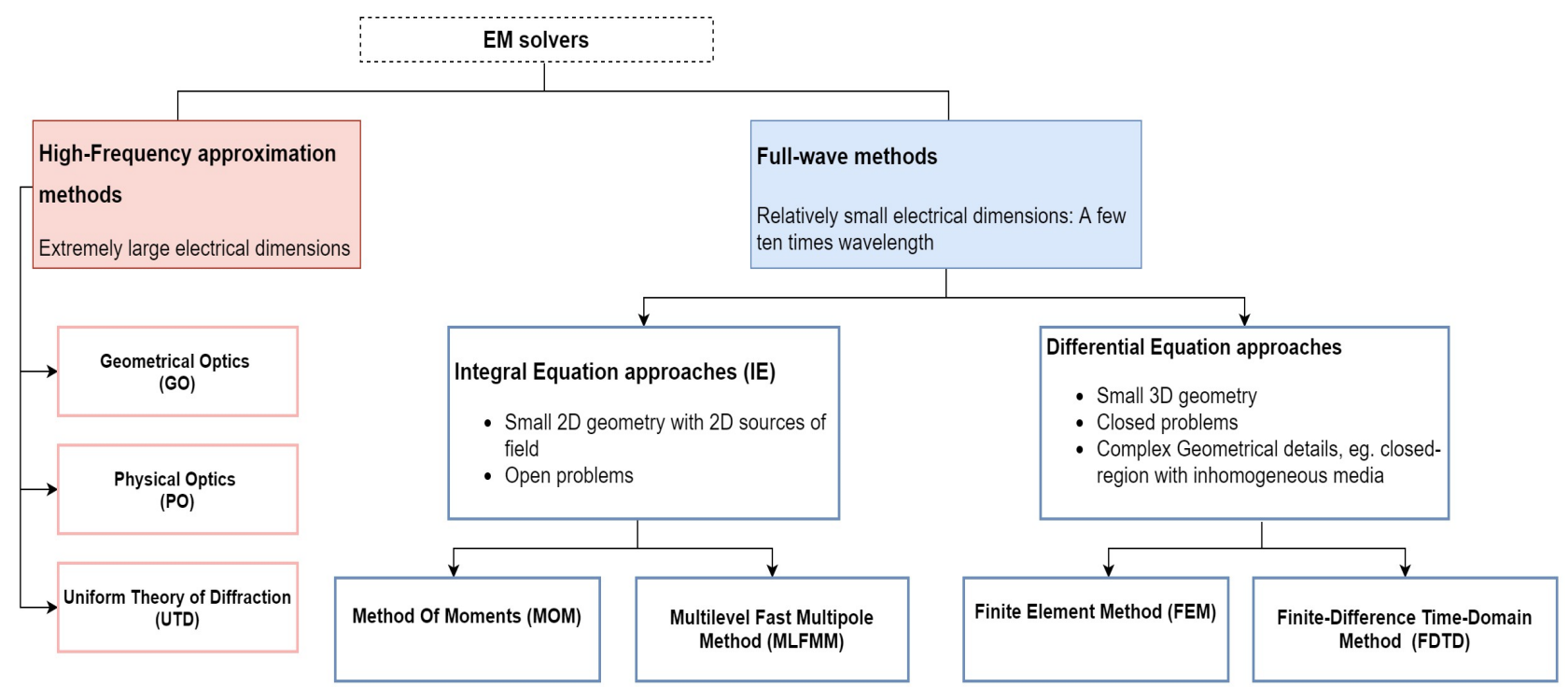

Figure 1. CEM solving methods classification

\section{Resonating behavior}

- Bounded/Unbounded (open boundaries)

As for the problem geometry sizes, one should answer the following question: "is the simulation space electrically large, much larger than a wavelength?". For antennas with extremely large electrical dimensions, High-Frequency approximation techniques are often used. They rely on the concept of rays to model the field behavior. High-Frequency techniques include geometrical optics (GO), physical optics may be used. It is based on the multi-pole extension technique; in contrast to MOM method, MLFMM computes the interactions of logarithmically subdivided groups of the Basis functions, hence reducing the matrix generated and decreasing computation time and memory complexity [22]. Combined with MOM method, it becomes highly preferable to analyze relatively more complex and larger structures like reflectors or antenna arrays. 
Table III. pros and cons of CEM solving methods

\begin{tabular}{|c|c|c|c|}
\hline $\begin{array}{l}\text { CEM solving } \\
\text { methods }\end{array}$ & MOM & FEM & FDTD \\
\hline Advantages & $\begin{aligned} &+ \text { Frequency domain } \\
&+ \text { 2D homogeneous structures } \\
& \text { such as thin wire and } \\
& \text { homogeneous planar layered } \\
& \text { antenna. } \\
&+ \text { Frequency sweep responses } \\
&+ \text { Adequate for open problems; } \\
& \text { No discretization of the } \\
& \text { surrounding space, only the } \\
& \text { antenna element, resulting in a } \\
& \text { smaller number of mesh } \\
& \text { elements. } \\
&+ \text { Accurate solution by directly } \\
& \text { solving the integral equations } \\
& \text { using Green's function } \\
& \text { approach } \\
&+ \text { Combined with acceleration } \\
& \text { technics such as Fast } \\
& \text { Multipole Method, enables } \\
& \text { analysis of electrically large } \\
& \text { structures. }\end{aligned}$ & $\begin{array}{l}+ \text { Frequency domain } \\
+ \text { Adequate for arbitrary 3D } \\
\text { geometries with curved or } \\
\text { slanted boundaries. } \\
+ \text { Better fit for narrow band } \\
\text { problems } \\
+ \text { Better fit for problems with } \\
\text { multiport } \\
+\quad \text { Very simple and accurate } \\
\text { handling of problems with } \\
\text { dispersive materials. } \\
+\quad \text { Use of multiscale modeling } \\
\text { in unstructured mesh; } \\
\text { therefore, refining mesh only } \\
\text { in relevant regions is made } \\
\text { easy. }\end{array}$ & $\begin{aligned} &+ \text { Time domain } \\
&+ \text { Best fit for problems with } \\
& \text { transient nature; Wide-band } \\
& \text { problems } \\
&+\quad \text { Adequate for radiation } \\
& \text { penetrating problems } \\
&+\quad \text { Easy and fast modeling of 3D } \\
& \text { regular geometries and } \\
& \text { inhomogeneous problems with } \\
& \text { multiple dielectric layers (in } \\
& \text { one simulation run) } \\
&+\quad \text { Better for parallel computation }\end{aligned}$ \\
\hline Disadvantages & $\begin{array}{l}\text { - Unsuitable for inhomogeneous } \\
\text { complex geometries. } \\
\text { - } \text { Inadequate for dielectrics with } \\
\text { non-linearity. } \\
\text { - Unsuitable for time varying } \\
\text { problems and wideband } \\
\text { applications } \\
\text { - More complex implementation } \\
\text { than DE methods. } \\
\text { - Inadequate for radiation } \\
\text { penetrating problems }\end{array}$ & 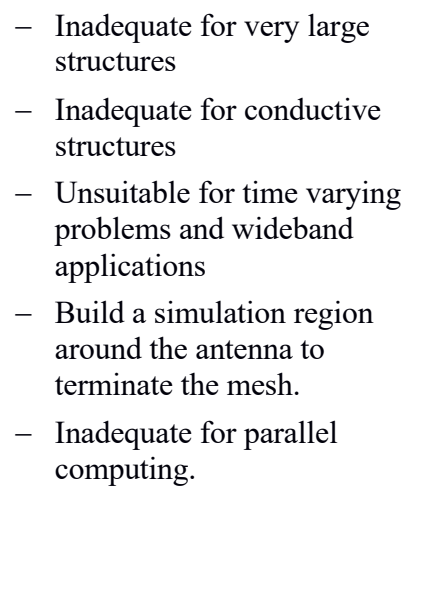 & $\begin{array}{l}\text { - Inadequate for multiport } \\
\text { problems } \\
\text { _ } \text { Build a simulation region } \\
\text { around the antenna (free space } \\
\text { discretization + absorbing } \\
\text { boundary conditions defined) } \\
\text { to terminate the mesh. } \\
\text { - } \\
\text { Truncation errors at each step. } \\
\text { - } \\
\text { Inflexible meshing; limited to } \\
\text { structured mesh in its classical } \\
\text { formula, therefore, not suitable } \\
\text { for geometries with extreme } \\
\text { irregularities. } \\
\text { - } \\
\text { Inadequate for problems with } \\
\text { dispersive materials. }\end{array}$ \\
\hline
\end{tabular}

On the other hand, differential techniques require placing the antenna in a 'box' to truncate the space and define the meshing domain in which the electromagnetic fields reside [23]. The resultant matrix to be solved is large but sparse; thus, only memory and CPU of $\mathrm{O}(\mathrm{n})$ time is required, with $\mathrm{n}$ is the number of discrete elements. Because the number of unknowns is proportional to the problem volume and meshing resolution considered, differential equation methods are particularly suitable for modeling relatively small three-dimensional volumes with complex geometrical details, for instance closed-region problems with inhomogeneous media [24]. Intrinsically, differential equations are not appropriate for large open problems, mainly because a discretization of the entire space under consideration is required; this space is limited in case of closed problems but corresponds to infinite space in case of open problems [25]. The most basic methods used are the Finite Element (FEM) and Finite Difference Time Domain (FDTD) methods.

FEM method is a frequency-domain solver which is used for narrow-band analysis of arbitrary shaped 3D antenna where fields are explicitly solved throughout entire volume. It also enables time efficient analysis of problems with multiple ports. Conversely, FDTD method is a time-domain solver which uses a time stepping algorithm. The latter updates the field values across the mesh cell time-step by time-step. The computational effort is proportional to the number of time steps; intrinsically, it depends on the frequency content of the signal. The wider the spectrum, the shorter the simulation time. Thereby, FDTD technique is 
more efficient for wide-band applications. Besides, FDTD lends itself extremely well to parallelization [23]. On the other hand, analyzing antenna design with $\mathrm{N}$ ports using FDTD would require $\mathrm{N}$ simulation runs, hence, it is inadequate for analyzing multi-port designs. Finally, in practice, FDTD is commonly used early on during simulation process to get quick answers "for any radiation and scattering problem" where "great accuracy is not the primary concern, and quite large runtimes and memory usage are acceptable" [26].

As a workaround to their limitations, hybridization methods have been proposed. Hybridization techniques result from combining two or more CEM solving methods into a single code. MOM-PO, FDTD-PO, FEM-FDTD, and FEM/MOM are examples of hybridization techniques commonly used in antenna simulation and analysis. Theses technics have been addressed in a recent review of literature on CEM methods [27]. Throughout discussion of hybrid techniques capabilities and limitations is out of the scope of this paper.

In summary, there is no perfect method that is suitable to model all types of antenna problems. Therefore, given the antenna problem and design goals, one should properly select the CEM solver. Based on the analysis above, we propose adequate CEM solvers for each antenna type reviewed in section 2 in an attempt to guide practitioners.

Wire antenna include monopoles, dipoles, helices, etc. For thin wires (i.e. Diameter $\leq$ wavelength/1000), MOM method is the perfect method to use; high accuracy is easily provided. Otherwise, in case of thick wire antenna structures (i.e. $\lambda / 1000<$ Diameter $\leq \lambda / 100$ ), FDTD is preferred then.

Reflector antennas are typically challenging to simulate because of the large computational resource required. For this type of problems MLFMM yields accurate results. If the reflector is extremely large, $\mathrm{MOM} / \mathrm{PO}$ is then recommended [27]. Another solution to consider is 'model decomposition'; the feed of the reflector is simulated separately, preferably using MOM method, and the outputs are used to represent it as an equivalent excitation source of the reflector.

Planar antennas range from simple patch antennas to sophisticated designs with complex feed structures and multiple dielectric layers. Therefore, several solvers might be considered. The MOM, hybrid FEM/MOM and FDTD/PO, MLFMM and FDTD solvers are all well suited, the best choice will depend on the characteristics of the antenna and capabilities of each solver. MOM solver is adequate for finite metal objects or slots in the presence of infinite, planar media. This enables the efficient analysis of complex microstrip antennas.

Array antennas are a set of antenna elements spaced and oriented according to a specific pattern to achieve a desired radiation pattern. Therefore, not only the characteristics of the individual elements should be considered, but also their coupling. In addition to the aforementioned CEM methods used for solving large problems, special formulations are available for simulating arrays such as: Periodic boundary conditions (PBC) for large periodic array geometries and special infinite multi-layered MOM for planar antenna arrays.

In case of wideband antenna, FDTD is the most appropriate since the full frequency spectrum is captured within a single simulation run.

\section{B. CEM SOFTWARE: SELECTION CRITERIA AND TRADE-OFFS}

Several CEM software have emerged over the last few decades. Table IV lists the most common ones along with their important characteristics. In principal, any CEM general purposes software could be used since they provide excellent mutual agreement even for challenging antenna structures [24] [22]. However, there are good practical reasons and factors to consider when deciding which software is best suitable to the application at hand. These factors are the following:

Fact $n^{\circ} 1$. Software support of the adequate solver given the problem modeling needs and objectives; this shall limit the range of software to consider.

Fact $n^{\circ} 2$. Open source vs commercial software; understand license type and cost for commercial ones.

Fact $n^{\circ} 3$. Technical requirements of the software - i.e., platforms compatibility, memory storage, GPU requirements, and support of parallel computing - to properly choose the software considering the user's needs and budget.

Fact $n^{\circ} 4$. Software documentation and training options availability.

Fact $n^{\circ} 5$. Software support for $2 \mathrm{D} / 3 \mathrm{D}$ geometry creation:

- Easy 2D/3D sketcher with parametric capabilities

- Availability of a material properties database

- Support of major CAD software formats importing/exporting

- Level of difficulty to grasp the software knowhow and to validate and run the model properly; is it required to be an expert to run it?

Fact $n^{\circ} 6$. Mesh generation and control; automatic or manual? What mesh advanced techniques are supported?

Fact $n^{\circ}$. Supported boundary conditions (e.g. ABCs, PMLs) for open problems

Fact $n^{\circ} 8$. Scripting and automation support.

Fact $n^{\circ}$. Support of post-processing capabilities, namely optimizers and parameter sweeps.

Fact $n^{\circ} 10$. Speed and accuracy.

As reported previously, selecting the adequate CEM solving method requires a clear definition of the problem 
Table IV. Benchmark of existing open-source and commercial CEM Software

\begin{tabular}{|c|c|c|c|c|c|c|}
\hline $\begin{array}{l}\text { Software } \\
\text { package }\end{array}$ & Platforms & License & GUI & 3D & Mesher & Solver \\
\hline $\mathrm{NEC}$ & $\begin{array}{l}\text { Windows/ } \\
\text { Linux }\end{array}$ & Open source & Partially & Yes & Manual & MOM \\
\hline MEEP MIT & Linux/ MacOS & Open source & --- & --- & --- & FDTD \\
\hline OpenEMS & $\begin{array}{l}\text { Windows/ } \\
\text { Linux }\end{array}$ & Open source & Yes & No & Manual & FDTD \\
\hline ELMER FEM & $\begin{array}{l}\text { Windows/ } \\
\text { Linux }\end{array}$ & Open source & Yes & Yes & Manual & FEM \\
\hline Momentum & $\begin{array}{l}\text { Windows/ } \\
\text { Linux }\end{array}$ & Commercial & Yes & Yes & Equidistant & MOM \\
\hline HFSS & $\begin{array}{l}\text { Windows/ } \\
\text { Linux }\end{array}$ & Commercial & Yes & Yes & Adaptive & $\begin{array}{c}\text { *FEM/ MOM/ FEBI/ Eigen Mode/ } \\
\text { SBR+/ Hybrid }\end{array}$ \\
\hline $\mathrm{CST}$ & $\begin{array}{l}\text { Windows/ } \\
\text { Linux }\end{array}$ & Commercial & Yes & Yes & $\begin{array}{c}\text { Adaptive/ } \\
\text { Manual }\end{array}$ & $\begin{array}{c}\text { *FIT/ MOM/ FEM/ Eigen Mode/ } \\
\text { Hybrid }\end{array}$ \\
\hline $\mathrm{xFdtd}$ & $\begin{array}{l}\text { Windows/ } \\
\text { Linux }\end{array}$ & Commercial & Yes & Yes & Adaptive & FDTD \\
\hline $\begin{array}{l}\text { COMSOL } \\
\text { multiphysics }\end{array}$ & $\begin{array}{l}\text { Windows/ } \\
\text { Linux }\end{array}$ & Commercial & Yes & Yes & Adaptive & *FEM/ Ray tracing \\
\hline FEKO & $\begin{array}{l}\text { Windows/ } \\
\text { Linux }\end{array}$ & Commercial & Yes & Yes & $\begin{array}{c}\text { Adaptive/ } \\
\text { Manual }\end{array}$ & *MOM/ FEM/ FDTD/ MLFMM \\
\hline WIPL-D & $\begin{array}{l}\text { Windows/ } \\
\text { Linux }\end{array}$ & Commercial & Yes & Yes & $\begin{array}{c}\text { Adaptive/ } \\
\text { Manual }\end{array}$ & MOM \\
\hline IE3D & $\begin{array}{l}\text { Windows/ } \\
\text { Linux }\end{array}$ & Commercial & Yes & Yes & --- & --- \\
\hline $\begin{array}{l}\text { EMPIRE } \\
\text { software }\end{array}$ & $\begin{array}{l}\text { Windows/ } \\
\text { Linux }\end{array}$ & Commercial & Yes & Yes & $\begin{array}{c}\text { Adaptive/ } \\
\text { Manual }\end{array}$ & FDTD \\
\hline $\begin{array}{l}\text { New Fasant } \\
\text { Soft. }\end{array}$ & $\begin{array}{l}\text { Windows/ } \\
\text { Linux }\end{array}$ & Commercial & Yes & Yes & $\begin{array}{c}\text { Adaptive/ } \\
\text { Manual }\end{array}$ & MOM \\
\hline SEMCAD & $\begin{array}{l}\text { Windows/ } \\
\text { Linux }\end{array}$ & Commercial & Yes & Yes & Adaptive & FDTD \\
\hline
\end{tabular}

*Software main Solver

nature in term of complexity, geometry sizes and type (2D, 2D-axisymmetric, 3D). A comprehensive discussion of capabilities of fundamental CEM solvers was presented previously in section 3.1 to help engineers make rational choices depending on the antenna subject to analysis. After that, one should define the license type of the software to use - open source or commercial - while considering the technical requirements of each software in respect of the user's needs and budget. From Table IV, we distinguish commercial and open source software. Academic/In-house CEM software also exist; however, they are often special purpose software for a specific antenna geometry, and their use is restricted to the institution's research community where they were developed; hence, they are out of the scope of this paper. To help practitioners make a reasonable choice, Table V highlights strengths and drawbacks of commercial and open-source software. Besides, [28] has made a comparative study between some popular commercial and open-source packages in term of speed and accuracy to clarify some misconceptions about open source CEM software and provide a proper knowledge of the existing open-source software capabilities.

Regardless of the software type selected, open-source or commercial, one should also investigate availability of documentation and training options; documentation, trainings and even community support are crucial to mitigate steep learning curves when using CEM software. Indeed, bad user experience due to documentation and support unavailability might greatly hinder software diffusion despite its computational capabilities, and thus it should not be neglected.

When it comes to antenna modeling efficiency and speed using CEM software, it depends greatly on the nature of the antenna problem. Whenever possible, identify the most timeconsuming tasks: Is it modeling and geometry creation, processing, or post-processing and optimization? Required time span to create and validate an antenna model is usually lengthy, and it is often underestimated especially for complex 
Table V. Pros and cons of open-source vs. commercial software packages for electromagnetic simulation derived from [28]

\begin{tabular}{|c|c|c|}
\hline & Commercial software & Open-source software \\
\hline Strengths & $\begin{array}{l}\text { - Complete and easy-to-use GUI } \\
\text { - Integrated pre-processing capabilities } \\
\text { - Possibility of importing/exporting data in } \\
\text { different file formats } \\
\text { - Various solvers are usually available, to be } \\
\text { chosen based on the kind of problem } \\
\text { - Solver engines can frequently exploit most of the } \\
\text { hardware capabilities } \\
\text { - A programming language is frequently } \\
\text { integrated with the package for complex } \\
\text { processing/modeling tasks } \\
\text { - Parametric optimizers, for selecting the best } \\
\text { solution with respect to the design specifications } \\
\text { - Post-processing software, capable of easily } \\
\text { generating graphs and images } \\
\text { - Extended documentation, user manuals, } \\
\text { simulation examples and tutorials } \\
\text { - Qualified assistance by the producer for the } \\
\text { solution of specific problems }\end{array}$ & $\begin{array}{l}\text { - No license costs and can be installed in } \\
\text { various workstations and in different versions } \\
\text { - Availability of the source code: in principle, } \\
\text { programs can be modified or customized with user- } \\
\text { specific functions } \\
\text { - The possible integration with other software } \\
\text { packages or tools is a typical feature of open- } \\
\text { source products } \\
\text { - Many sophisticated post-processing tools can be } \\
\text { employed } \\
\text { - The formats of files adopted in the program flow } \\
\text { are known, and standard import/export formats } \\
\text { are sometimes supported } \\
\text { - Developers' communities are often very available } \\
\text { for discussions and suggestions }\end{array}$ \\
\hline Weaknesses & $\begin{array}{l}\text { - High license fees and each computer may need } \\
\text { its own license (e.g., node-locked licenses) } \\
\text { - Each functionality/module of the software is } \\
\text { usually sold separately, and the use of } \\
\text { graphical processing units (GPUs) and/or } \\
\text { parallel computing often have additional fees } \\
\text { - Difficult integration of the package with other } \\
\text { external software products } \\
\text { - File formats are often proprietary and not } \\
\text { documented. This makes input/output file } \\
\text { manipulations arduous with external tools }\end{array}$ & $\begin{array}{l}\text { - GUI is often absent } \\
\text { - There are very few reliable and user-friendly solver } \\
\text { engines, their complexity results in a very steep } \\
\text { learning curve } \\
\text { - Any software program implements only a single } \\
\text { numerical solving method } \\
\text { - Each phase of the design usually requires some or } \\
\text { more different software packages, which should be } \\
\text { integrated by the user } \\
\text { - Powerful but difficult to use geometric modeling } \\
\text { modules and mesh generators } \\
\text { - The capability to interface and manage some } \\
\text { proprietary file formats (adopted in commercial } \\
\text { packages) is absent even in high-quality products } \\
\text { - The generation of graphical results and parametric } \\
\text { optimization are possible only by means of external } \\
\text { tools (that should be integrated by the user) } \\
\text { - Documentation is sometimes incomplete, obsolete, } \\
\text { or completely absent }\end{array}$ \\
\hline
\end{tabular}

designs used in custom applications where predefined CAD model are usually inexistent. When the CEM software is not user-friendly and requires an expert to run it, or when it lacks built-in geometry sketcher, CAD import/export tools or a material properties database, antenna geometry creation and modeling might become laborious and time-consuming; even if the execution time takes many hours, it is easily eclipsed by several days or even weeks spent on model creation and validation. Furthermore, this might also lead to flawed models and inaccurate results. Therefore, at this level, it is crucial to evaluate beforehand how simple it is to create the geometric model using the built-in tools of the selected CEM software.

On the other hand, sometimes optimization and parametric analysis of a relatively simple model might take weeks. At this level, simulation execution time becomes a key element. Enhancement of physical parameter sweeps using fully parametric solvers, in conjunction with postprocessing and automation tools support, enables efficient model optimization in the least amount of time. Moreover, defining model accuracy requirements and addressing solution convergence will facilitate definition of both meshing resolution and method (structured/unstructured, adaptive/manual) and problem boundary conditions. Inadequate meshing or boundary conditions might alter the antenna model characteristics, and thus it will lead to lengthy simulations or erroneous results. Further discussions of the impact of meshing methods and boundary conditions on results accuracy along with major sources of simulation errors are presented in the next section. Practical design tips to mitigate these issues are also highlighted. 
Finally, it is worth mentioning that correct modeling and simulation lead to compatible results. This has been thoroughly investigated in the literature to clarify any ambiguity about results discrepancies when modeling a problem using several tools [24, 29]. Indeed, various studies of commercial software have been published in the literature to define their pros and cons for a wide range of applications. However, the findings were very user dependent. This was even more accentuated for tricky complex antenna geometries, where performance is very sensitive to meshing conditions or ground-plane size, or where excitation modeling is critical. As a result, the inconsistency between the various tools raised many doubts about the validity of the choices made during simulations and questioned the performance of certain tools. To address the previous issues, a benchmark was carried out in 2014 by EurAAP Software Working Group. Several leader software vendors were directly involved, namely Agilent, with the ADS-Momentum and the EM-Pro software, Ansoft-Ansys with the HFSS software, CST with the CST Studio software tools. They were asked to perform the simulations, under the credo "a software vendor is the most optimal user of its own tool" [30]. The aim was to investigate whether an efficient design flow would reduce discrepancies between tools. Analysis of the exact same antenna structure was conducted, with the complete connector topology provided. This was achieved by issuing to all vendors a reference 3D CAD "sat" file of the complete structure with the feed and connector set-up. An important implication of this benchmark is that if two qualified candidate software or more has been considered for the analysis of a given problem, they shall provide compatible results as long as the simulation is conducted correctly.

\section{CUBESAT ANTENNA DESIGN USING CEM TOOLS: WORKFLOW AND SOURCES OF ERRORS}

During early design phases of a Cubesat, chosen antenna system must be simulated to evaluate not only radiation characteristics such as gain and return losses, but also the effects of the satellite body structure and co-located components such as solar panels. Therefore, the optimal antenna configuration can be defined, and radiation pattern can be enhanced by means of CEM simulation tools. In general, antenna design is a multi-phased process, and it is reiterated until optimal results are generated, and a preliminary documentation of the antenna model could be defined. As depicted in Fig. 2, three major phases in the design process of antennas can be identified: pre-processing phase, solution phase, and post-processing phase.

Pre-processing phase: After choosing the Cubesat antenna type, initial design parameters are defined in a preliminary specifications document; these parameters might include resonating frequency, bandwidth, dimensions, materials used, etc. The next step is approximating the antenna problem by creating a valid computer model. This task can be in turn broken down into various subtasks. First, the initial simulation setup is established; design parameters and simulation units are defined as per design equations given in the literature for that particular antenna. Next, numerical solver is chosen, excitation sources, boundary conditions and frequency sweep ranges are defined. Then, a geometric 3D model of the antenna is developed considering the predefined design parameters. Finally, generation of the input data for the solver engine. To carry out these tasks, the user can interact with the software either with a graphical user interface (GUI) or by means of script files.

Solution phase: In order to solve the electromagnetic problem, the antenna model is first approximated using structured or unstructured mesh depending on the nature of the problem in hand - i.e. is it regular or irregular. Structured regular mesh consists of squares and hexahedral and follows a uniform pattern, while unstructured mesh consists of triangular and tetrahedral cells for $2 \mathrm{D}$ and 3D structures, respectively. Further discussion of un/structured meshing is provided later in this section. Then, data generated is supplied to a solver engine. The solving loop will stop when convergence is achieved given chosen boundary conditions.

Post-processing phase: At this stage, user requested data - such as return loss, VSWR, 2D/3D gain, and directivity can be extracted from the numerical solver output files. Generated results are then verified and validated against design goals and specifications. If the results are not optimal, parametric studies and optimization analysis (local or global) of the problem are carried to produce an optimal antenna design. This task can be almost effortless if automated optimization of multi-variable and multi-goal problems, while using various algorithms, such as Genetic Algorithm (GA) and particle swarm, is supported by the CEM software.

The generated results will serve different purposes; they may be used to perform performance comparisons with other existing antenna solutions, to numerically validate the antenna model against known analytical results or simulation results generated by other solving methods, or even to generate input data for other simulations purposes. Another important objective is to finalize antenna design documentation necessary for the antenna prototyping and development.

Inaccuracy or disagreement of simulation results with known analytical results, simulation results generated by other solving methods, or testing findings are common issues encountered during numerical validation of the antenna model [24] [22] [29, 31] These issues are attributable to the following factors:

- Poor antenna model approximations

- Inadequate frequency sweeping range input

- Feed modeling

- CEM solving algorithm capabilities and limitations

- Inadequate boundary conditions

- Inappropriate Meshing resolution/method 


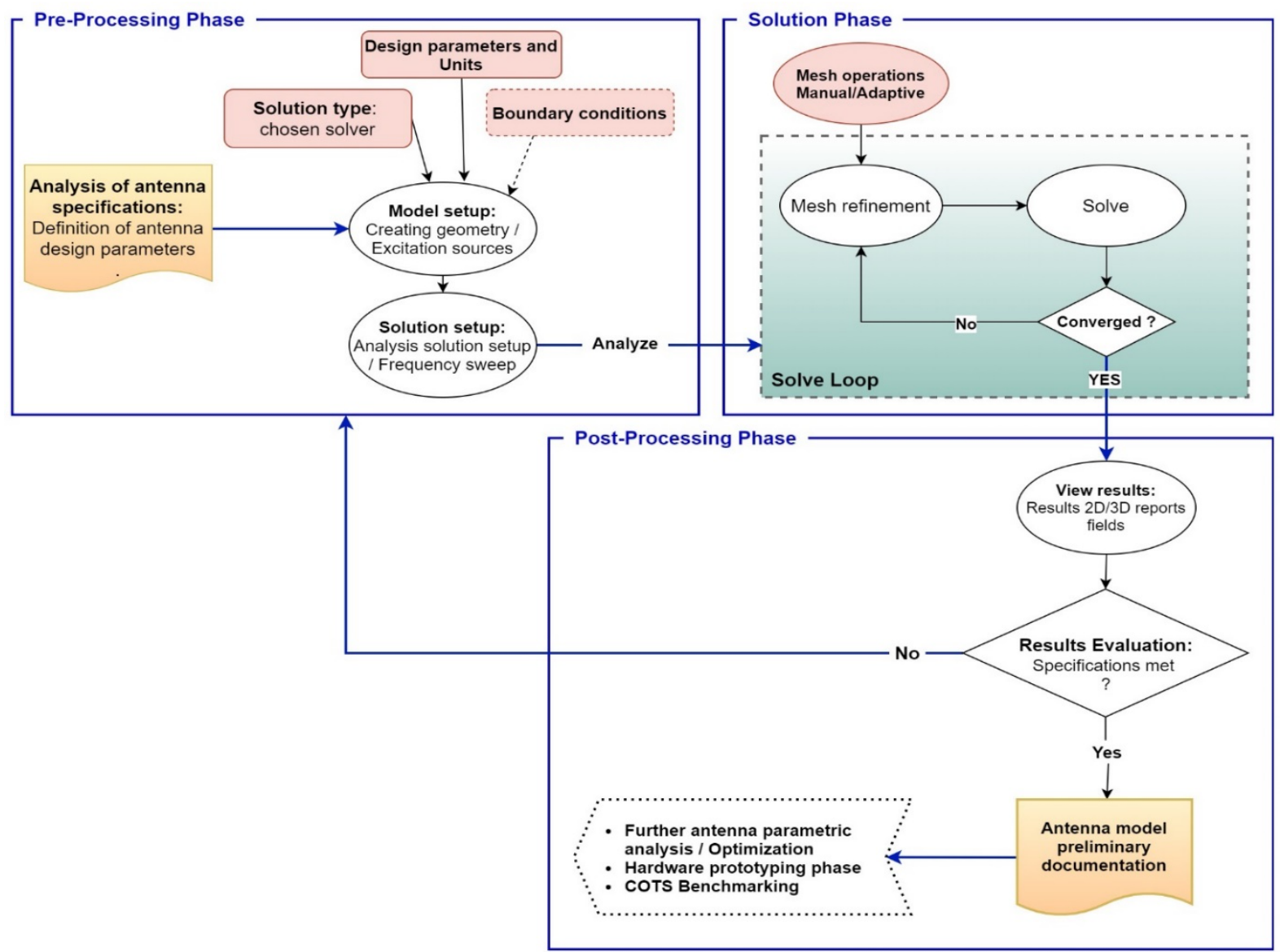

Figure 2. Antenna design workflow using CEM tools

Poor antenna model approximation might result either from solving overly simplified governing equations, forsaking losses modeling, or overlooking some critical aspects of the model such as finite dielectric layers. Invalid model parameters' input is another reason for antenna model inaccuracy. When it comes to antenna feed modeling using CEM software, it is often idealized; the connector and transmission line are forgotten in purely simulation environments and are far from the pure ideal $50 \mathrm{Ohm}$ condition. Besides, the antenna feed model differs from one software to another. This is a possible reason for discrepancies of simulation results between the various tools.

On the other hand, inappropriate implementation of boundary conditions shall induce simulation errors. This is especially true for unbounded problems, such as antenna radiation and scattering problems in free space. Solving Maxwell equations numerically for this type of problems using Integral Equation (IE) methods is relatively straightforward, and the fields can be calculated anywhere in space during results post-processing using near-field to farfield transformation. However, when using Differential Equation (DE) methods, truncation of the computational grid domain is required to limit simulation infinite space to finite volume and reduce model complexity, hence, significantly decreasing solution time and computing resources requirements. Now, the key question is: how to perform "transparent" truncation of the simulation domain without producing unphysical artifacts due to boundary reflection? In the literature, various formulations of boundary conditions have been defined to address the previous question. An elaborate discussion of boundary conditions is beyond the scope of this paper. However, we will present their main features to help engineers make rational decisions, and hence decrease simulation errors.

The choice of the adequate formulation is dictated by the requirements that a boundary condition must satisfy with respect to (i) media types and (ii) the location of the source of the field [31]; is it going to be placed far from the source or in its vicinity? The Perfect Electric Conductor (PEC) is a simple example of boundary conditions placed in the vicinity of the source. PECs are used to specify boundaries that behave as perfect electric conductors; thus, they might serve as peripherals of waveguides, metallic surfaces of an antenna or infinite ground-plane for PCB boards. PECs are perfectly reflecting allowing no energy to escape the simulation domain along that boundary. It is used to mimic the behavior of metals for very high frequencies where the skin depth is extremely small and irrelevant. Another example is the 
Perfectly Magnetic Conductor (PMC). It is the magnetic equivalent of the PEC. It is not physical but merely an artifact. Both PECs and PMCs are often used as symmetric walls for symmetric structures with symmetric wave propagation in order to reduce the volume of the computational domain. Choosing between a PEC or a PMC is often tricky. To make the right choice one should consider the source polarization. As for periodic structure, if the electromagnetic field is also periodic, a Periodic Boundary Conditions (PBC) is the best option because it allows direct simulation of the periodic system by simulating only a single unit cell or element [32]. To model problems involving field calculation in an infinite domain such antenna radiation and scattering problems, Absorbing Boundary Conditions (ABC) and Perfect Matching Layers (PML) are used. Arbitrarily defining a boundary condition at a finite distance from the antenna induces reflections of the outgoing waves toward the domain of simulation, hence adding a spurious field to the solution. ABCs and PML both mimic infinite extension of the computational domain by absorbing the radiated outgoing fields without any inward reflections, thus providing good approximations of infinite domain calculations. In practice, when modeling radiation and scattering problems where the incident wave is normal or near normal to the boundary and the wave medium is homogeneous at the grid boundaries, it is recommended to use $\mathrm{ABCs}$ because they are faster to process and easier to setup than PMLs. Moreover, they can be assigned to an arbitrary shaped boundary. However, if the fields are propagating at non-normal incidence to the boundary, an $\mathrm{ABC}$ yields inaccurate results. In this case, using a PML is more appropriate [32] since PMLs performance is independent from the wave angle of incidence.

Finally, applying a "good" mesh is crucial; a mesh is efficient if it ensures a good compromise between simulation speed and accuracy. Meshing consists in spatially discretizing the model geometry into finite elements using either structured or unstructured cells. Structured regular mesh consists of squares and hexahedral, while unstructured mesh consists of triangular and tetrahedral cells for 2D and 3D structures, respectively. In practice, increasing the number of mesh cells enables capturing the field gradients in an accurate manner, hence increasing results accuracy. However, this also increases the simulation time, and the computational resources required to simulate the problem becomes prohibitive. Thereby, one might consider increasing the size of the mesh cell to speed up the simulation time, and hence increasing the time step [33]; nevertheless, the mesh size cannot be increased arbitrarily because it is constrained by the following limiting factors:

- When using FDTD solvers, the time step should be small enough to verify "courant stability condition", given by the following equation considering a hexahedral mesh cell, with $\Delta \mathrm{x}, \Delta \mathrm{y}$ and $\Delta \mathrm{z}$ mesh step in each direction:

$$
\Delta t_{\max } \leq \frac{1}{U_{\max }}\left[\frac{1}{\Delta x^{2}}+\frac{1}{\Delta y^{2}}+\frac{1}{\Delta z^{2}}\right]^{-\frac{1}{2}}
$$

With $U_{\max }$ is the maximum wave velocity within the model, and it is material properties dependent.

- Numerical dispersion: Each frequency component propagates in the mesh at a different velocity. So, to mitigate this issue, the smallest mesh size should be in the order of $\lambda 10$.

- The mesh cell should be small enough to represent the smallest feature in the model critical areas.

In summary, from the previous discussion, it is clear that any inaccuracy or disagreement of simulation results are intrinsically related to a lack of knowledge of the problem nature or a gap in the antenna design knowhow when using CEM tools. to mitigate the aforementioned issues, we suggest some recommendations and practical tips to follow during antenna design and analysis:

From simplified to full model: Start with a simplified model, when possible, to gain insights into both physical and computational aspects of the solution. Once the initial model is validated, it is then easier to design the complete model. This transition, from simple to a complete model, might include going from 2D to 3D model, from linear to nonlinear materials, from lossless to lossy materials and from coarse to fine meshing. It is worth mentioning that it is recommended to use PEC when modeling metallic structures of antennas operating in very high frequencies to simplify the model. Indeed, as it was mentioned previously, PEC is used to mimic the behavior of metals for very high frequencies where the skin depth is extremely small and irrelevant. On the other hand, nowadays, there are some support software tools which enables fast and efficient antenna design and modeling, such as Antenna Magus. It provides good starting designs tuned to the specifications of the application. It also increases design efficiency by helping engineers make informed choices during antenna design. Developed antenna models can then be exported to CEM software such as FEKO or CST for 3D simulation and postprocessing to ensure antenna model integration into its target environment.

$\checkmark$ Pay great attention to geometry details, namely small features and sharp corners. They might lead to field singularities and overly fine mesh, hence increasing simulation duration and memory requirements. Therefore, it is better to omit sharp corners or any other small feature if they are not critical to the problem or if they are less than $\lambda / 10$; include only necessary details to create an accurate model. Another way to deal with these, 
and still can be retained in a model, is coarse meshing if they have no influence on the field solutions; otherwise, rounding up sharp corners is a better alternative to create more realistic simulation models.

\section{Accord great importance to boundary conditions type} for unbounded problems. Also, perform a convergence study to evaluate truncation errors at the boundary and define accuracy order beforehand. For time-dependent calculations, truncation errors appear as dispersion. This can be mitigated through local mesh refinements.

\section{$\checkmark$ Choose the right meshing method/approximation for} better accuracy: Automatic meshing, using structured or unstructured cells, greatly simplifies the meshing process of an antenna model. For simple cases, the brute-force approach of "automesh-and-run" is often enough and provides quick valid results; thus, further mesh refinement is unnecessary. However, for complex antenna geometries mesh refinement is usually needed; Using structured mesh cells for complex structures often yields inaccurate results [34]. In practice, when modeling complex designs, the brute-force approach is usually used to gain initial insights into the solution nature. It is also used to provide a priori knowledge about model critical components and regions with strong field gradients. At this point, depending on the chosen CEM software capabilities, two approaches are possible: (i) Use unstructured adaptive meshing for the entire model altogether, or (ii) use structured local meshing refinement in conjunction with some advanced techniques, such as Perfect Boundary Approximation (PBA), which is supported in CST software, instead of using the traditional staircase approximation. The latter induces errors when meshing arbitrarily curved geometries. Besides, it doesn't efficiently handle mesh cells at the boundary of two different materials or more: in case two different materials were present in a single mesh cell, it is resolved by filling the cell with the material that occupies the highest ratio. Another special meshing method worth mentioning is the Multilevel Sub-gridding Scheme (MSS). It was developed to expand the capabilities of structured mesh methods to model small features or antennas inside large computational volumes by locally refining the cell-size using sub-grids.

Unstructured mesh accurately complex models with curved or slanted geometries containing materials with high dielectric-contrast mainly because it applies no preestablished mesh patterns; So, it enables elegant scaling from tiny to large cells and vice-versa [34]. This flexibility is not readily available when using structured meshing. The latter requires further local meshing for selective mesh refining of critical regions where more accuracy is required, while relaxing the mesh for irrelevant areas. However, the process can be tricky for non-specialists. It might also be lengthy, especially when model remeshing is required after parametric studies or due to geometry modifications. Another solution among others to simplify meshing of complex geometries is by partitioning the geometry, then perform automatic meshing for each partition. Antenna partitioning is commonly applied to reflector antenna, where the feed is analyzed separately, then the resulting field is applied to the reflector structure.

$\checkmark$ Increase results accuracy and decrease processing time by choosing the right mesh resolution: As it was mentioned previously, the minimum resolution required for antenna radiation problems is about 10 elements per wavelength. Increasing the number of cells results in (i) a considerable increase in memory requirement and (ii) a decrease of simulation time step, thus the total simulation time will increase. Furthermore, the medium nature and the model's size and critical parts impose practical constraints on mesh size and indirectly mesh number of cells for volume-based numerical methods. For instance, extremely fine details in a model induce considerable numerical challenges because they restrict meshing resolution, thus overly fine mesh is used for the complete model.

$\checkmark$ Optimize the antenna design while considering target working environment and collocated components: an antenna is affected by everything around it, especially metallic structures. The radiated electromagnetic fields from an antenna interact with nearby materials, thus resulting in a change of the antenna resonant frequency of operation and its impedance mismatch. In fact, A poorly matched antenna shall degrade the link budget margin by 10 to $30 \mathrm{~dB}$ and severely impair the communication link. Therefore, the antenna must be designed and integrated considering its end surrounding environment. This is a key point to consider when developing an antenna system for a reliable Cubesat communication link.

\section{CASE STUDY: DESIGN AND ANALYSIS OF A HALF- WAVE DIPOLE FOR MASAT-1 MISSION}

Masat-1 is a 1Unit Cube-satellite with a maximum weight of $1.33 \mathrm{~kg}$ and measuring $10 \times 10 \times 10$ centimeters (Fig. 3). Cubesats are a standardized format of nanosatellites created in 1999 to ease access to space at a minimum cost and development time. Masat-1 shall operate in the UHF band at a resonant frequency of $437.5 \mathrm{MHz}$. In the remaining of this section, we shall present rationales behind the choice of the antenna configuration for Masat-1 mission and decisions made during the design the process in respect of the guidelines provided previously in this paper.

Setting up a reliable TTC link between Masat- 1 and the ground station implies providing good link margins. This could be achieved by, among others, compensating pointing 
losses and polarization changes due to the Faraday rotation. Indeed, on the one hand, Masat-1 antenna system should be able to radiate and receive signals in the assigned band regardless of the spacecraft nadir pointing accuracy and the attitude control system performance. It is therefore highly recommended to use omnidirectional antennas. On the other hand, Faraday rotation phenomena might result in sever misalignments of ground and space antennas, especially when a linear-linear configuration is used [35]; thus, it is preferable to use circular polarization at least at one end of the link. Only during the initial stages of the project, it was decided to use passive attitude control for Masat-1. Hence, it was quite difficult to accurately predict the orientation of the satellite. So, using circular polarization with the same handedness in both sides for lower polarization loss was out of question; instead, we decided to use an omnidirectional antenna with linear polarization at the CubeSat end. After reviewing past literature, two antenna configurations were considered because they verified most of Masat-1 mission requirements and constraints: monopole and dipole antennas. They both have near omnidirectional radiation pattern. However, the dipole was selected since its performance is independent of any ground plane; unlike the monopole, a half-wave dipole antenna design is quite straightforward.

** Source : https://alen.space/basic-guide-nanosatellites/

Figure 3. Common standard Cubesat units

As per Masat-1 philosophy, we intend to use COTS
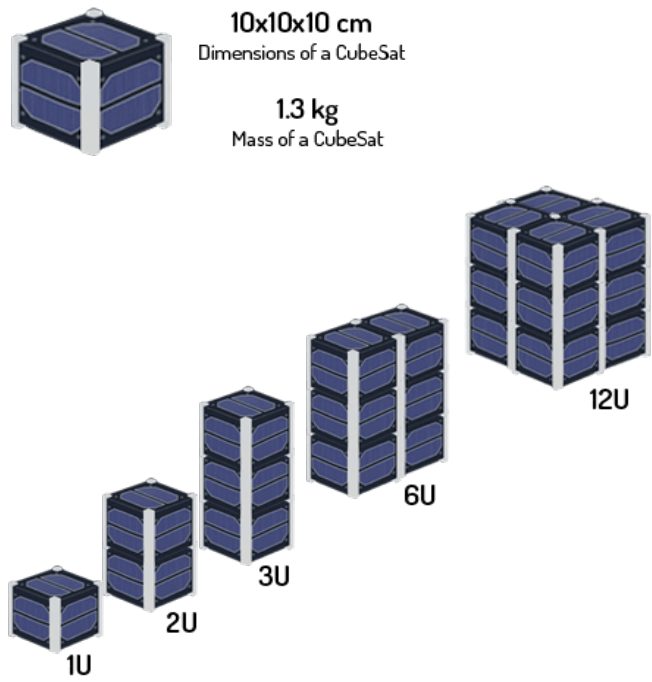

products to decrease development time and cost [35] [36]. Benchmarking existing COTS solutions, we selected a COTS dipole antenna from ISIS- Innovative solutions in space company. Nevertheless, we developed a 3D model of a halfwave dipole not only to generate an initial specification document for Masat-1 antenna system, but also to gain deeper understanding of the half-wave dipole antenna design process and practical constraints. Eventually, this model shall provide the basis for custom design of in-house antenna system for subsequent missions.

To calculate the half-wave dipole design parameters, the following equation is used:

$$
\mathrm{L}=0.5 \mathrm{~K} . \mathrm{c} / f
$$

With $\mathrm{K}$ is the $\mathrm{K}$-factor that illustrates the correlation between the dipole length and its diameter. For thick wire antenna, i.e. $\lambda / 1000<2 R \leq \lambda / 100$, the K-factor is $\sim 0.95$. Therefore, the simplified equations for the half-wave dipole antenna are the following, as in [37]:

Where:

$$
\begin{gathered}
\mathrm{L}=143 / f \\
\lambda=\mathrm{c} / f \\
\mathrm{Gap}=\mathrm{L} / 200 \\
\mathrm{R}=\lambda / 1000
\end{gathered}
$$

As mentioned previously, another crucial factor to consider for a reliable of a TTC link for Masat-1 is the effect of the antenna surrounding environment. Indeed, Masat-1 metallic structure can be detrimental to the antenna performance and will change its frequency of operation and impedance. This is why Masat- 1 antenna must be tuned properly after including the metallic Cubesat structure in its final design.

According to the discussion reported in section 3.1, FEM method is unsuited for efficient modeling of wire antennas and conductive structures, and MOM might provide poor results when analyzing thick or $3 \mathrm{D}$ conductive structures. Hence, to analyze the half-wave dipole model proposed for Masat-1, assess the effect of the satellite structure on the antenna performance and tune the antenna model accordingly, we selected FDTD as a solving method. Thereby, we selected CST Microwave Studio software because it is best known for its transient solver Finite Integration Technique (FIT), a relative of FDTD method. Even though it is a commercial software, CST is a generalpurpose software, user-friendly, and supports efficient parametric analysis and optimization tools. We analyzed the half-wave dipole antenna in CST following these steps; first, a 3D CAD model of the antenna has been developed using the parameters defined in Table VI.

Table VI. Calculated parameters of Masat-1 half-wave dipole initial model

\begin{tabular}{cc}
\hline Parameters & Value \\
\hline Resonant frequency & $437.50 \mathrm{MHz}$ \\
\hline
\end{tabular}




\begin{tabular}{cc}
\hline Wavelength $(\lambda)$ & $685.71 \mathrm{~mm}$ \\
\hline Dipole length L & $326.85 \mathrm{~mm}$ \\
\hline Dipole radius R & $0.68 \mathrm{~mm}$ \\
\hline Dipole feeding gap & $1.63 \mathrm{~mm}$ \\
\hline
\end{tabular}

The antenna material is aluminum. CST provides a large database for materials. It also provides the option to define new materials if necessary. Next, the feeding point of the antenna was assigned; the antenna is fed by a $50 \Omega$ discrete port, y-directed and excited with a Gaussian waveform. Proper port disposition, from antenna transmitting element to the antenna ground element, is crucial to ensure accurate results. Then, the frequency sweep range was fixed to 300 $600 \mathrm{MHz}$. This range should be wide enough to decrease simulation time since CST transient solver is more efficient for wideband applications. CST automatically built a boundary box around the antenna to limit the simulation domain using adequate PML (open add) conditions. This is to simulate the halfwave dipole performance in free space. Then, the simulation domain is discretized using adaptive structured meshing. Fig.4 illustrates the dipole antenna at the center of this region without the spacecraft metallic structure, hence, evaluating the antenna radiation performance in free space conditions. If the aforementioned pre-processing tasks were carried out correctly, the return loss (S11) should be less than $-10 \mathrm{~dB}$ for the antenna operating bandwidth. Ideally, we aim to achieve an operating bandwidth ranging between 430 and $440 \mathrm{MHz}$. Moreover, the VSWR ratio should be less than 1.5 which connotes a good antenna matching to the input impedance.

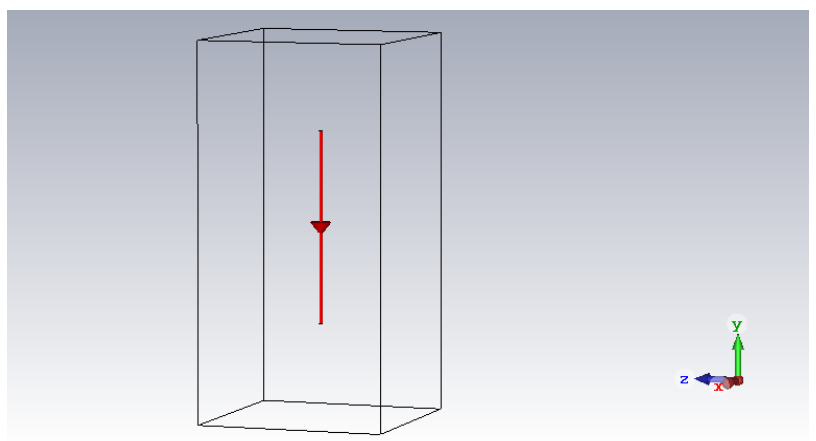

Figure 4, A half-wave dipole antenna with its bounding box in CST

The initial model of the dipole yielded inaccurate results in term of frequency. As shown in Fig. 5(a), the operating frequency of the antenna given the initial model parameters is different than $437.5 \mathrm{MHz}$, however, a good bandwidth and impedance matching were achieved. Thereby, a first optimization was carried out to tune the antenna resonant frequency. Given equation 2, tuning a dipole antenna frequency lies in adjusting either its length or radius while fixing the other parameters. For completeness of the study, the simulation was carried out also for various values of the gap to take into consideration its effect on the results.
This provided initial insights on what to expect when adjusting the length, the radius or the gap. Thus, subsequent parametric and optimization analysis are carried out in an easy and timely manner.
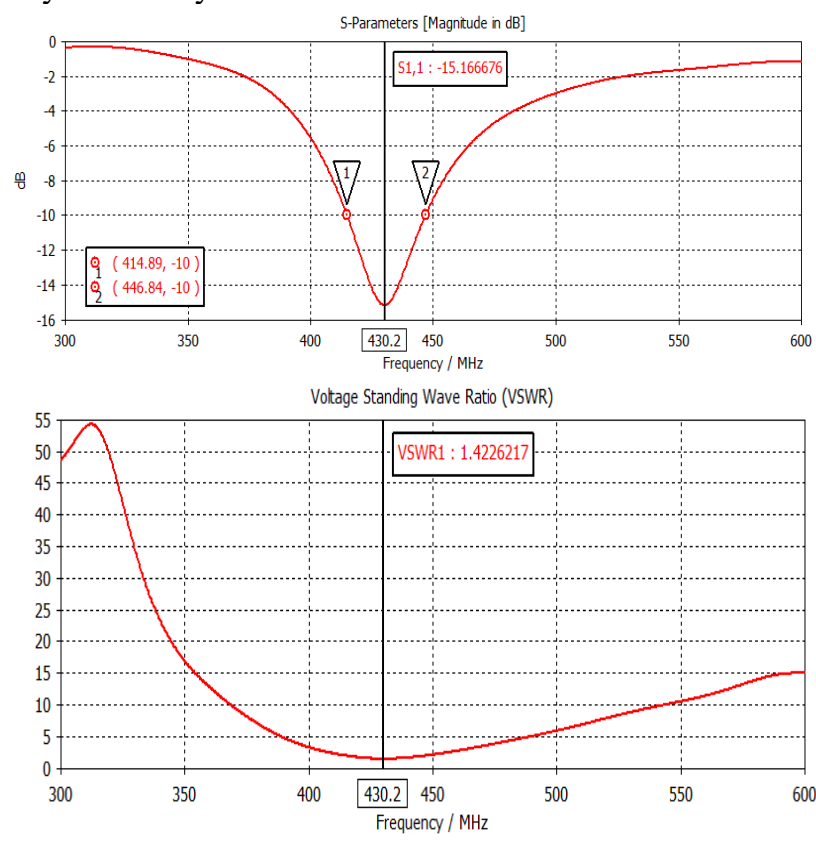

Figures $5(a)$ and $5(b)$. VSWR and return loss S11 of a half-wave dipole using the parameters defined in Table III
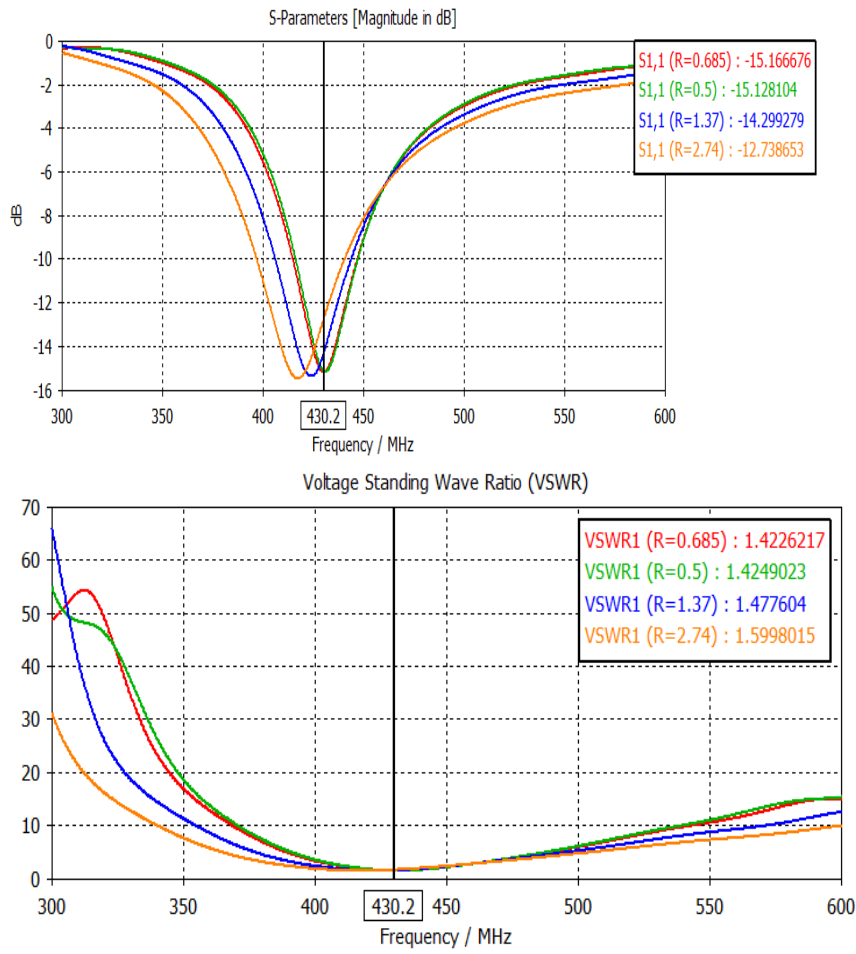

Figures 6(a) and 6(b). S11 and VSWR of a simple half-wave dipole for different radius values with $L=326.85 \mathrm{~mm}$ and $G a p=1.63 \mathrm{~mm}$

Fig. 6(a) and 6(b) depict the correlation between the dipole radius and its operating frequency; by increasing the 
radius, the frequency is decreasing, and the bandwidth is getting wider. This is feature is especially useful to design a broadband dipole antenna. However, the dipole should not be too thick to preserve good VSWR ratio (VSWR $<1.5$ ). On the other hand, Fig. 7(a) and 7(b) depict how decreasing the gap to some extent enhances the VSWR but at the cost of increasing the resonant frequency. In practice, it is well known that the gap shouldn't be too small to avoid sparking between the dipole elements. Nonetheless, it cannot be arbitrarily increased as it should be less than $\lambda / 2$. Finally, Fig. 8(a) and (b) supports findings in the literature, that is the higher the operating frequency the shorter is the dipole.
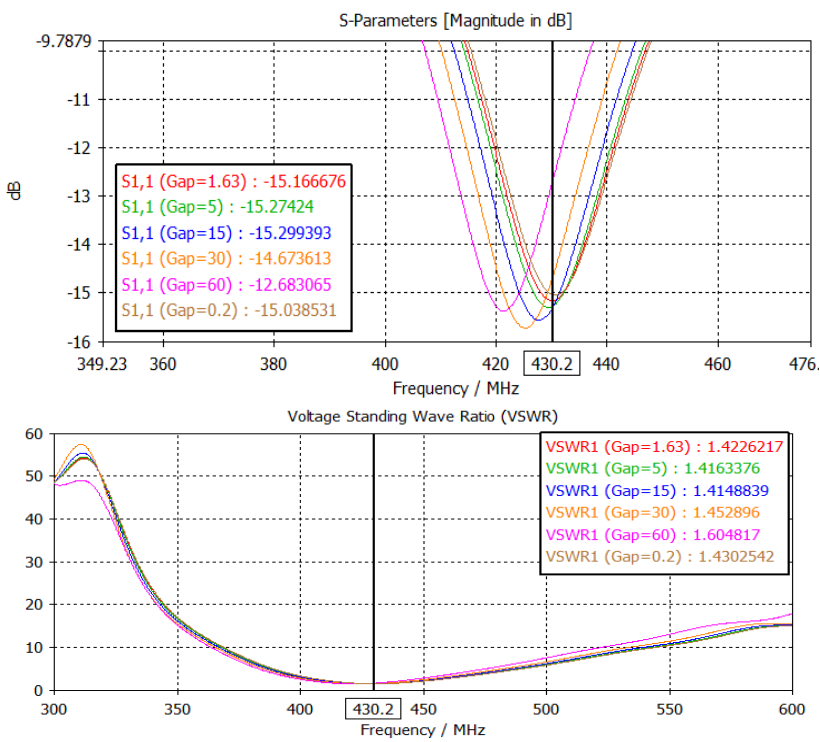

Figures $7(a)$ and $7(b)$. A simple half-wave dipole performance for different feeding gap effect with $L=326.85 \mathrm{~mm}$ and Radius $=0.685 \mathrm{~mm}$
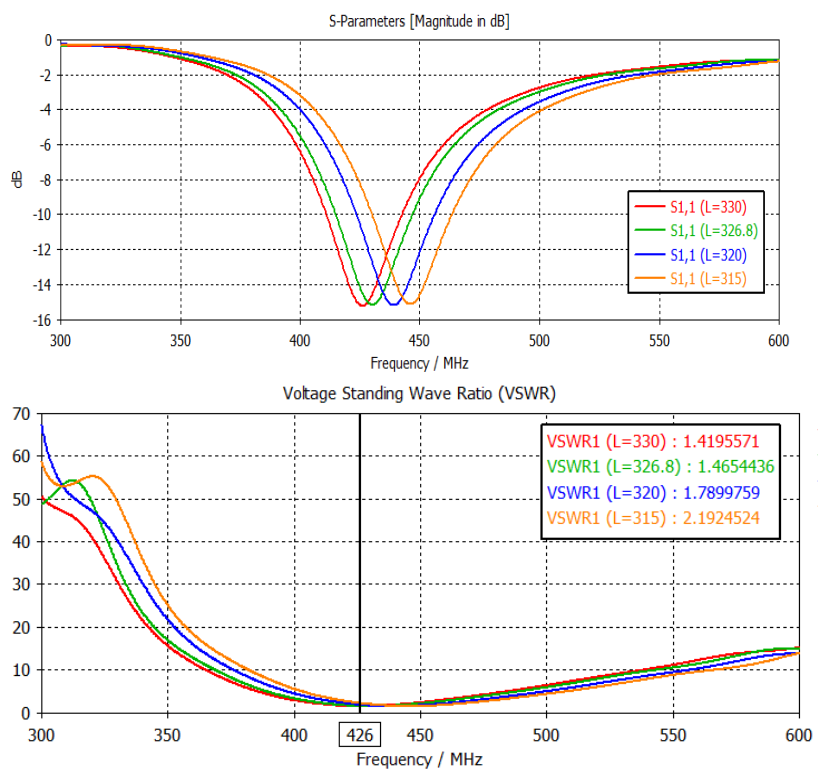

Figures 8 (a) and 8 (b). A simple half-wave dipole performance for different lengths with Gap $=1.63 \mathrm{~mm}$ and dipole Radius $=0.685 \mathrm{~mm}$.
In summary to design a half-wave dipole antenna, we propose the following based on the previous findings: After defining (i) the optimal radius then (ii) the appropriate gap that yield the best VSWR results and provides enough bandwidth for our application, any further tuning of the dipole antenna resonant frequency is better done by altering only the length of the antenna. This concurs with what J. Carr suggests in [38]; he points out that "there is only one proper way to tune a dipole antenna: adjust the length of the antenna elements".

In our case, to optimize the initial model of the halfdipole, the following decisions were made. we kept the initial radius because it yielded a good bandwidth; the gap was defined; Then, the length was shortened to tune the operating frequency to $437.5 \mathrm{MHz}$. Consequently, the optimal model of Masat-1 dipole antenna in free space was achieved using the following parameters:

$\mathrm{L}=321.148 \mathrm{~mm}(\sim 0.47 \lambda)$, Gap $=2 \mathrm{~mm}$ and Radius $=$ $0.685 \mathrm{~mm}$.

After that, the antenna was integrated with the Cubesat structure, however, the operating frequency of the dipole antenna shifted to $435.29 \mathrm{MHz}$, as it is illustrated in Fig. 10 . Therefore, another optimization analysis is necessary. It is important to note that an insulating layer of non-conducting material was added on top of the metallic structure as it is depicted in Fig. 9 for simulation purposes. This is to prevent grounding the input signal fed to the antenna poles, and hence nullifying the antenna overall radiation. A simple PCB material commonly used in antenna design such as FR-4 or RT-5880 can be used for the insulating layer. At first glance, one might consider using FR-4 for the following reasons: it is readily available; it has good reproductivity; and it is cheap. However, it is a lossy dielectric; variations in its electrical permittivity results in dramatically shifts of the resonant frequency, and its high loss tangent significantly affects the antenna radiation efficiency. Moreover, these losses are even more evident in higher frequencies. Therefore, we selected RT-5880 because it exhibits better results.

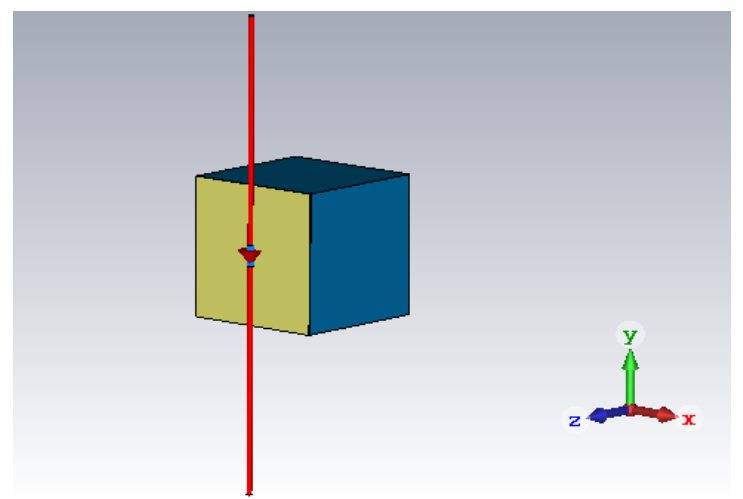

Figure 9. Masat-1 half-wave dipole integrated with the cubic structure in CST 


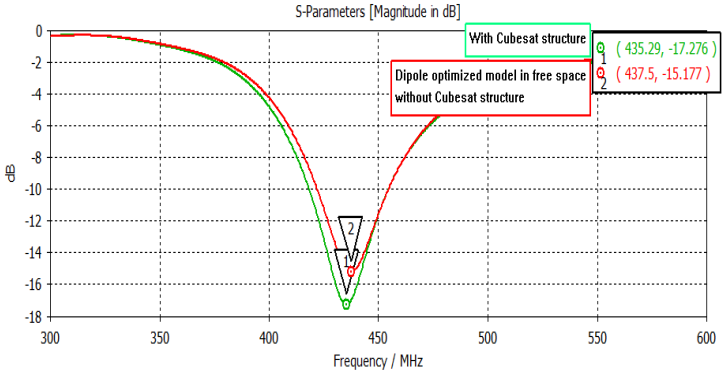

Figure 10. Masat-1 halfwave dipole antenna return loss before and after introducing the Cubesat metallic structure

Tuning the antenna frequency required further shortening of the dipole length. We got excellent results given the following parameters: $\mathrm{L}=3.148 \mathrm{~mm}$, Gap $=2 \mathrm{~mm}$ and Radius $=0.685 \mathrm{~mm}-$ Fig. $11(\mathrm{a})$ and 11(b). Indeed, the value of return loss has been found as $-17.29 \mathrm{~dB}$, VSWR $=1.3$ was achieved, and $36.4 \mathrm{MHz}$ of bandwidth is readily available. Also, as it is shown in Fig. 12(a) and 12(b), the directivity of the antenna model obtained is $2.12 \mathrm{dBi}$ which is almost identical to the theoretical ones [38].
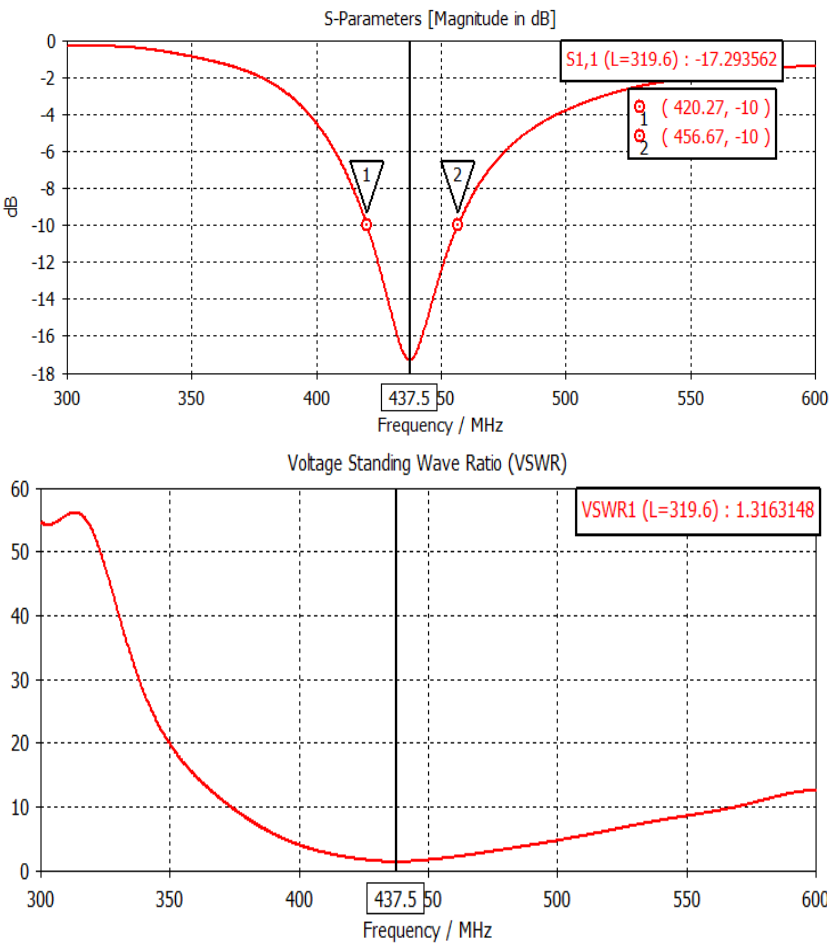

Figure 11(a) and 11(b). VSWR and S11 plots for the optimized model of the half-wave dipole antenna with the metallic structure integrated
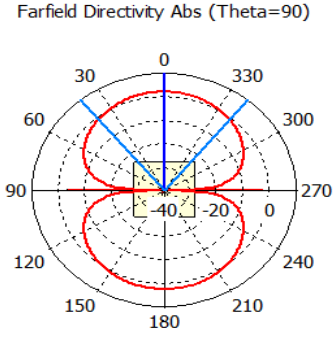

Phi / Degree vs. dBi

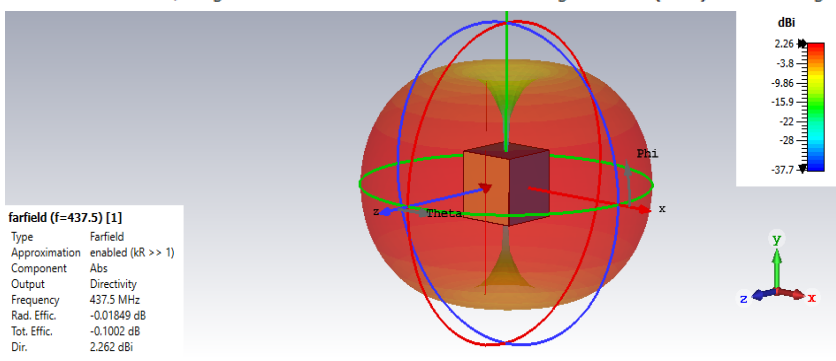

Figure 12(a) and 12(b). Polar and 3D radiation pattern plots for the optimized model of the half-wave dipole antenna with the metallic structure integrated

\section{CONCLUSION}

Antenna system design for Cubesat spacecraft is trending toward "smaller, smarter, cheaper, and faster" [1]. To achieve this, efficient design flow coupled with powerful CEM tools is needed. This paper attempts to highlight important factors to consider when choosing the adequate CEM tool/software for analysis of a Cubesat antenna model. It also emphasizes design considerations to take to reduce simulation errors.

Depending on gain requirements, antenna types used for various applications in Cubesat communications have been shortly described. A review of different CEM solving techniques is presented leading to the following guidelines: the first major consideration is the antenna geometry; is it 'planar' in nature or a 'genuine' 3D structure? For planar structures like thin wires and homogeneous planar layered antennas, MOM method is recommended. However, it is not applicable for thick wires or larger, non-linear and inhomogeneous planar structures. As an alternative MLFMM is considered. It is an improved and faster version of MOM, and it is also used to simulate finite, larger complex antennas like reflectors and finite size arrays. FEM and FDTD are mainly used for 3D arbitrarily shaped antennas. Whilst FEM is a frequency-domain solver used for narrow-band applications, FDTD is a time-domain method considered for wide-band analysis. FEM is faster than FDTD for simulation with a large number of ports. Nonetheless, FDTD lends itself extremely well to parallelization unlike MOM and FEM, which resolves to hybridization techniques for simulation 'acceleration'. It is worth mentioning that FDTD method is commonly used early on during simulation process to get quick answers "for any radiation and scattering problem" where "great accuracy is not the primary concern, and quite large runtimes and memory usage are acceptable" [26]. 
This paper also highlights the major factors to consider when selecting the adequate CEM solving methods and software packages with the belief that a proper knowledge of the existing CEM tools capabilities and limitations is very useful for skilled practitioners and non-specialist alike to make rationalized choices during the design process. Furthermore, the general workflow of antenna modeling and analysis using these tools has been described. Also, issues such as erroneous/inaccurate simulation results, results which take excessively long to compute, or discrepancies found between various CEM software were investigated. The findings indicate that a poor antenna model approximation, inadequate frequency sweeping range input, incorrect feed modeling, lack of knowledge of CEM solving algorithm capabilities and limitations, and most importantly using inadequate boundary conditions or inappropriate meshing resolution/method are the major causes for these issues. Thereby, practical design tips and recommendations were provided. Finally, the case study of half-wave dipole antenna for Masat-1 mission was presented as a practical example, in which some of the aforementioned tips and guidelines were applied, and satisfying results were obtained.

\section{FUNDING STATEMENTS}

This is work received no external funding.

\section{REFERENCES}

[1] S. Gao, Y. Rahmat-Samii, R. E. Hodges and X.-X. Yang, "Advanced antennas for small satellites," Proceedings of the IEEE, vol. 106, no. 3,, pp. 391-403,, March 2018.

[2] J. Costantine, Y. Tawk, A. Ernest and C. Christod, "Deployable antennas for CubeSat and space communications," Proc. European Conf. Antennas and Propagation, p. 837-840, 2012.

[3] P. Muri, O. Challa and J. McNair, "Enhancing small satellite communication through effective antenna system design," in Proc. Military Communications Conf. (MILCOM),, p. 347-352., 2010.

[4] E. Pittella, . S. Pisa and A. Nascetti, "Design of an antenna system for CubeSat satellites," Proc. 2nd Int. Academy Astronautics Conf. University Satellites Missions and CubeSat Winter Workshop, 2013.

[5] H. L. Abdul, J. S. Ping, N. A. Saidatul, L. Herwansyah, S. K. Podilchak, C. Suramate, F. J. Mohd, A. A.-H. Azremi, A. Prayoot and G. Steven, "A review of antennas for Picosatellite applications," Hindawi International Journal of Antennas and Propagation, $\mathrm{p}$. 17, 2017.

[6] P. A. Warren, . J. W. Steinbeck, . R. . J. Minelli and C. Mueller, "Large, deployable S-band antenna for a $6 \mathrm{U}$ CubeSat," Proc. 31st Annu. AIAA/USU Conf. Small Satellites (SSC), Logan, UT, USA, august 2015.
[7] Y. Rahmat-Samii and R. . L. Haupt, , "Reflector antenna developments: a perspective on the past, present, and future," IEEE Antennas and Propagation Magazine, vol. 57, no. 2, p. 85-95., Oct 2015.

[8] R. E. Hodges, N. E. Chahat, D. J. Hoppe and J. D. Vacchione, "The Mars Cube One deployable high gain antenna," Proc. IEEE Int. Symp. Antennas and Propagation (APSURSI), p. 1533-1534., 2016.

[9] N. Chahat, R. E. Hodges, . J. Sauder, M. Thomson, E. Peral and . Y. Rahmat-Samii, "CubeSat deployable Kaband mesh reflector antenna development for earth science missions," IEEE Trans. Antennas Propag., vol. 64, no. 6, p. 2083-2093, June 2016.

[10] V. Manohar and . Y. Rahmat-Samii, "Characterization of Ka-band mesh surfaces for CubeSat reflector antennas: From simple wire grid model to complex knits," Proc. Int. Union Radio Sci. Nat. Radio Science Meeting, pp. 1-2, 2016.

[11] R.-S. Yahya, M. Vignesh and M. ,. Joshua, "For satellite, think small, dream big: a review of recent antenna developments for Cubesats," IEEE Antenna and propagation magazine, vol. 59, no. 2, April 2017.

[12] R. . E. Hodges, M. . J. Radway, . A. Toorian and D. . J. Hopp, "ISARA-integrated solar array and reflectarray CubeSat deployable Ka-band antenna," Proc. IEEE Int. Symp. Antennas and Propagation, p. 2141-2142, 2015.

[13] J. M. Kovitz, V. Manohar and Y. Rahmat-Samii, "Feed horn optimization using feed+reflector co-simulation for advanced reflector antennas," in International Applied Computational Electromagnetics Society Symposium - Italy (ACES), 2017.

[14] . S. . K. Sharma, Handbook of Reflector Antennas and Feed System,Volume1: theory of reflectors, Norwood, MA, USA: Artech House, 2013.

[15] F. E. Tubbal, R. Raad and K. W. Chin, "A survey and study of planar antennas for pico-satellites," IEEE Access, vol. 3, p. 2590-2612, 2015.

[16] T. Alam, . M. . T. Islam, M. . A. Ullah and . M. Cho, "A solar panel-integrated modified Planner Inverted F Antenna for low earth orbit remote sensing nanosatellite communication system," Sensors, vol. 8, p. 2018.

[17] G. . F. Kurnia, B. . S. Nugroho and A. . D. Prasetyo, "Planar Inverted-F Antenna (PIFA) array with circular polarization for nano satellite application," Proc. Int. Symp. Antennas and Propagation (ISAP), p. 431-432, 2014.

[18] X. Liu, J. Liu, D. R. Jackson, J. Chen, P. W. Fink and G. Y. Lin, " Broadband transparent circularly-polarized microstrip antennas for CubeSats," Proc. IEEE Int. Symp. Antennas and Propagation (APSURSI), p. 15451546, 2016. 
[19] S. Tariqk and R. Baktur, "Circularly polarized UHF upand-downlink antennas integrated with CubeSat solar panels," Proc. IEEE Int. Symp. Antennas and Propagation, p. 1424-1425, 2015.

[20] R. M. Rodriguez-Osorio and E. F. Ramirez, "A handson education project: Antenna design for inter-CubeSat communications," IEEE Antennas Propag.Mag., vol. 54, p. 211-224, 2012.

[21] J. Klein, J. Hawkins and D. Thorsen, "Improving CubeSat downlink capacity with active phased array antennas," Proc. IEEE Aerospace Conf., pp. 1-8, 2014.

[22] DeLisle and J.J., "what are differences between various EM simulation numerical methods?," Micowave and RF, November 2014.

[23] Keysight Technologies, "Which electromagnetic simulator should I use ?," 2012.

[24] G. A. E. Vandenbosch and A. Vasylchenko, "A Practical Guide to 3D Electromagnetic Software Tools," in Microstrip Antennas , IntechOpen, 2011.

[25] G. A. E. Vandenbosch, "Computational Electromagnetics in Plasmonics," in Plasmonics Principles and Applications, IntechOpen, October 2012.

[26] D. B. Davidson, Computational Electromagnetics for RF and Microwave Engineering, second edition, Cambridge Press,, 2011.

[27] P. Sumithra and D. Thiripurasundari, "A review on computational electromagnetics methods," Advanced Electromagnetics, vol. 6, no. 1, March 2017.

[28] A. Fedeli, C. Montecucco and G. L. Gragnani, "OpenSource Software for Electromagnetic Scattering Simulation: The Case of Antenna Design," Electronics, vol. 8, no. 12, 2019.

[29] D. Pozard, S. Duffy and N. Herscovici, "A comparison of commercial software packages for microstrip antenna strip," IEEE Antennas and Propagation Society International Symposium, 2000.

[30] G. A. E. Vandenbosch, "State-of-the-Art in Antenna Software Benchmarking: Are We There Yet?," IEEE Antennas and Propagation Magazine, vol. 56, no. 4, p. 300-308, 2014.

[31] J.-P. Berenger, Perfectly Matched Layer (PML) for Computational Electromagnetics, Morgan \& Claypool, 2007.

[32] W. Yu, Electromagnetic Simulation Techniques Based on the FDTD Method, Wiley, September 2009.

[33] "Understanding Time Domain Meshing in CST Microwave Studio," July 2010. [Online]. Available: https://www.researchgate.net/file.PostFileLoader.html? id $=578$ c450ceeae3937441b63a1\&assetKey $=$ AS\%3A38 $5033333428224 \% 401468810508239$.
[34] K. Sankaran, "Are you using the right tools in computational?," Engineering Reports Published by John Wiley \& Sons Ltd, September 2019.

[35] I. Latachi, M. Karim, A. Hanafi, T. Rachidi, S. Dahbi and S. Zouggar, "Link budget analysis for a LEO cubesat communication subsystem," Proceedings - 3rd International Conference on Advanced Technologies for Signal and Image Processing, ATSIP 2017, 2017.

[36] I. Latachi, T. Rachidi, M. Karim and A. Hanafi, "Building low cost CubeSat: Guidelines and design approach," Advances in the Astronautical Sciences, 2018.

[37] A. Osman, A. A. Yassin, B. Ali, H. Ahmed and S. Noor, "Design and simulation of high performance half wave-dipole," in International Conference on Computing, Control, Networking, Electronics and Embedded, 2015.

[38] J. J. Carr, Practical antenna handbook, Fourth edition: McGraw-Hill, 2001.

[39] D. Tayli, "Computational tools for antenna analysis and design," Doctoral dissertation (2018), Lund university, Lund, Sweeden., 2018.

[40] I. I. M. A. Sulayman, S. H. A. Almalki, M. S. Soliman and M. O. Dwairi, "A comparative study for designing and modeling patch antenna with different electromagnetic CAD approaches - A case study," Progress in Electromagnetic Research Sympos, 2016.

[41] M. Kupresak, X. Zheng, G. A. E. Vandenbosch and V. Moshchalkov, "Benchmarking of software tools for the characterization of nanoparticles," Optics Express, vol. 25 , no. $22,2017$.

[42] J. Hoffmann, C. Hafner, P. Leidenberger, J. Hesselbarth and S. Burger, "Comparison of electromagnetic field solvers for the $3 \mathrm{D}$ analysis of plasmonic nanoantennas," Modeling Aspects in Optical Metrology II, 2009.

[43] F. Shamshad and M. Amin, "Comparison simulation between HFSS, CST and WIPL-D for design of dipole, horn and parabolic reflector antenna," Advances in Computational Mathematics and its Applications (ACMA), vol. 1, no. 4, 2012.

[44] E. Miller, "Computational Electromagnetics," in The Electrical Engineering Handbook, Ed. Richard C. Dorf, Boca Raton: CRC Press LLC, 2000.

[45] T. Gonzalez, J. Diaz-Herrera and A. Tucker, Computing handbook: Computer Science and Software Engineering, CRC press, May 7, 2014. 